\title{
Dr. Franz Xaver Felberbauer, MSc, Chirurg
}

\author{
1967-2017
}

\author{
Johannes Miholic
}

Online publiziert: 15. Januar 2018

(C) Springer-Verlag GmbH Austria, ein Teil von Springer Nature 2018

Dr. Franz Felberbauer ist gefallen, hätte man früher gesagt. Wie die Toten einer älteren Generation ist er zu früh gegangen, ohne Abschied, und mit einer Plötzlichkeit, die uns Verbliebene ratlos und in Unverständnis allein gelassen hat. Er ist gescheitert auf seinem Lebensexperiment, auf der mutigen Entdeckungsreise, die manchen auf einen Zauberberg verführt, einem Reich von trial und error, dem Wagnis einer hohen Kunst, deren Eintrittspreis der beherzte Schnitt ist. Die Macht über Leben und Tod mag es auch sein, die Entdecker auf dieses „Eiland der Kirke“ lockt. Aber nicht nur Franz Felberbauer war nicht Odysseus genug, dort ungefährdet $\mathrm{zu}$ weilen (so der Zauberer im Zauberberg). Die Kraftkammer einer jungen, meist männlichen Meute auf der Suche nach Erkenntnis, Innovation, und Macht, den Tod zu besiegen, übersteht mitunter schlecht, wer zur Verformung nicht bereit, dem aufrechten Gang und seinem geraden Weg verpflichtet ist. Der Kampf gegen Krankheit und Tod ist letztlich eine Sisyphus-Aufgabe, wie Albert Camus sagte, eine Absurdität. Franz Felberbauer ist an dieser Absurdität zerbrochen. Selbst risk taker, ist er der Verführung einer Sucht erlegen.

Blutüberströmt wurde er mit tödlichen Verletzungen in jenes Haus, an seine Klinik gebracht, dessen universitärer Hintergrund ein Paradiesgärtlein der Kreativität sein sollte und oft ist. Aber wo Diversität, Freiheit und Erhellung bisweilen den Fortschritt des Wissens beflügelt, herrscht auch Hierarchie und Regulierung. Den Eintritt in die unsichere Laufbahn eines akademischen Arztes wagte Franz Felberbauer 1994 an der chirurgischen Klinik in Wien. Engagement für die Patienten, Hilfsbereitschaft, Zuverlässigkeit im Operationssaal und selbstbewusstes nonkonformisti-

Ao. Univ. Prof. Dr. J. Miholic $(\bowtie)$

Herrengasse 6-8, 1010 Wien, Österreich

johannes.miholic@meduniwien.ac.at sches Denken zeichneten ihn aus. Die Dissertation am Institut für Pathophysiologie und Allergieforschung, im renommierten Pflügers Archiv für Physiologie erschienen, „Fiberoptic measurement of intracellular pH in intact rat-liver using $p H$-sensitive dyes. “, erhält 9 Zitate in den folgenden 19 Jahren. Weitere Arbeiten betreffen die Physiologie der Leber und die Kinderchirurgie, stets auf höchstem Niveau. Auf der Allgemeinchirurgie widmet sich Franz Felberbauer dann dem rasch expandierenden Gebiet der bariatrischen Chirurgie. Aktive Teilnahme an Tagungen ergänzt eine beträchtliche Anzahl von Publikationen. Franz war ein beliebter und inspirierender Teamkollege in wissenschaftlichen Projekten. Sein Werk hat bis heute 74 Zitate als Erstautor und 751 als Koautor erhalten. In den 43 Publikationen des Science Citation Index nicht enthalten sind seine zwei letzten Schriften: „Gastric emptying and distal gastrectomy independently enhance postprandial GLP-1 release after a mixed meal and improve glycemic control in subjects having undergone pancreaticoduodenectomy." und „The relationship between postprandial cholecystokinin concentrations and $\left[{ }^{11} \mathrm{C}\right]$ methionine uptake of the pancreas in patients after pancreaticoduodenectomy. " erscheinen posthum.

Publish or perish - am Ende obsiegt das perish und der Wettlauf nach Erfolg endet im Absurden. Albert Camus: „Es gibt nur ein wirklich ernstes philosophisches Problem: den Selbstmord. Sich entscheiden, ob das Leben es wert ist, gelebt zu werden oder nicht, heißt auf die Grundfrage der Philosophie antworten." Aber: „Der Kampf gegen Gipfel vermag ein Menschenherz auszufüllen. Wir müssen uns Sisyphus als einen glücklichen Menschen vorstellen."

Franz, du fehlst. Du hattest entschieden Stil in deinem Leben und im Wirken an der Klinik und - verzeih - Stil in deinem Tod. Du wirst uns noch lange begleiten. 